\title{
The Drosophila DmGluRA is required for social interaction and memory
}

\section{Brian P. Schoenfeld ${ }^{1,2}$, Richard J. Choi ${ }^{1,2}$, Catherine H. Choi ${ }^{1,2,3,4}$, Allison M. Terlizzi ${ }^{1}$, Paul Hinchey ${ }^{1}$, Maria Kollaros ${ }^{1}$, Neal J. Ferrick ${ }^{1,2}$, Eric Koenigsberg ${ }^{1}$, David Ferreiro ${ }^{1}$, David A. Leibelt ${ }^{1}$, Steven J. Siegel ${ }^{5}$, Aaron J. Bell ${ }^{1,2}$, Thomas V. McDonald ${ }^{1}{ }^{*}$, Thomas A. Jongens ${ }^{2}{ }^{*}$ and Sean M. J. McBride ${ }^{1,2,5}$ *}

' Section of Molecular Cardiology, Department of Molecular Pharmacology and Medicine, Albert Einstein College of Medicine, Bronx, NY, USA

2 Department of Genetics, University of Pennsylvania School of Medicine, Philadelphia, PA, USA

${ }^{3}$ Department of Medicine, Lehigh Valley Health System, PA, USA

${ }^{4}$ Department of Dermatology, Drexel University College of Medicine, Philadelphia, PA, USA

${ }^{5}$ Department of Psychiatry, University of Pennsylvania School of Medicine, Philadelphia, PA, USA

\section{Edited by:}

Andrew Lawrence, Florey

Neuroscience Institutes, Australia

\section{Reviewed by:}

Daniela Brunner, PsychoGenics, USA

Charles D. Nichols, Louisiana State University Health Sciences Center,

USA

Terence Pang, Florey Institute of Neuroscience and Mental Health, Australia

\section{${ }^{*}$ Correspondence:}

Sean M. J. McBride, Department of Psychiatry, University of Pennsylvania School of Medicine, 3535 Market

Street, 2nd floor Room 2062

Psychiatry, Philadelphia, PA 19104

USA.

e-mail:smjmcbride@gmail.com;

Thomas V. McDonald, Section of

Molecular Cardiology, Department of Molecular Pharmacology and

Medicine, Albert Einstein College of Medicine, Forchheimer G35, 1300

Morris Park Avenue, Bronx, NY 10461, USA

e-mail: tom.mcdonald@einstein.yu.edu; Thomas A. Jongens, Department of Genetics, University of Pennsylvania School of Medicine, 538A Clinical Research Building, 415 Curie

Boulevard, Philadelphia, PA 19104,

USA

e-mail: jongens@mail.med.upenn.edu
Metabotropic glutamate receptors (mGluRs) have well-established roles in cognition and social behavior in mammals. Whether or not these roles have been conserved throughout evolution from invertebrate species is less clear. Mammals have eight mGluRs whereas Drosophila has a single DmGluRA, which has both $\mathrm{Gi}$ and $\mathrm{Gq}$ coupled signaling activity. We have utilized Drosophila to examine the role of DmGluRA in social behavior and various phases of memory. We have found that flies that are homozygous or heterozygous for loss of function mutations of DmGluRA have impaired social behavior in male Drosophila. Futhermore, flies that are heterozygous for loss of function mutations of DmGluRA have impaired learning during training, immediate-recall memory, short-term memory, and longterm memory as young adults. This work demonstrates a role for mGluR activity in both social behavior and memory in Drosophila.

Keywords: mGluR, memory, Drosophila, long-term memory, DmGluRA, learning

\section{INTRODUCTION}

The Metabotropic glutamate receptors (mGluRs) in mammals have been shown to be involved in memory formation, longterm depression (LTD), long-term potentiation in mammals and linked to autism spectrum disorders (ASDs) in humans (Serajee et al., 2003; Mukherjee and Manahan-Vaughan, 2012). In mammals there are eight mGluRs, which are divided into three groups. Canonically, previous studies have established that group I mGluRs in mammals activate the Gq pathway, while group II and group III mGluRs activate the Gi/Go signaling pathway (Niswender and Conn, 2010; Chaki et al., 2012; Mukherjee and Manahan-Vaughan, 2012). However, there is accumulating evidence that in mammals, due to promiscuity of coupling to Gi and Gq, group II mGluRs may activate the Gq signaling pathway and induce LTD in a manner dependent on phospholipase C (PLC) and inositol trisphosphate receptor (InsP3R) activity (Huang et al., 1997, 1999a,b; Otani et al., 1999, 2002), and group I mGluRs are capable of activating Gi (Kreibich et al., 2004). In Drosophila there is only one mGluR, DmGluRA, which is coupled to Gi and Gq signaling (McBride et al., 2005; Pan and Broadie, 2007; Pan et al., 2008; Gatto and Broadie, 2009; Tessier and Broadie, 2012). Gi coupled signaling is able to engage and activate both the PI3K and ERK signaling cascades as well as increase glycogen synthase kinase-3 beta (GSK-3beta) activity and Gq mediated mGluR activation is also able to activate GSK-3beta activity (Fan et al., 2004; Huang et al., 2006; Beaulieu et al., 2009; Yuskaitis et al., 2010). 
Drosophila DmGluRA expression has been demonstrated in the brain including expression in areas critically involved in shortterm memory such as the antennal lobes (ALs) and mushroom bodies (MBs; McBride etal., 1999; Zars et al., 2000; Yu et al., 2004; Pesavento et al., 2008) and long-term memory in the MBs (McBride et al., 1999; Pascual and Preat, 2001) where expression is particularly heavy (Ramaekers et al., 2001; Pan and Broadie, 2007; Pan etal., 2008). More recently a detailed analysis of DmGluRA protein expression in the central complex has been published, a region of the brain where the expression of several other metabotropic receptors implicated in mammalian learning was found (Kahsai etal., 2012). The MBs in the insect are thought to be analogous to the mammalian hippocampus as first postulated from structural similarity to the human hippocampus in 1850 by the French physiologist and anatomist DuJarin (Dujardin, 1850; Davis, 1993, 2011; Busto et al., 2010; Skoulakis and Grammenoudi, 2006). Additionally, DmGluRA protein has been demonstrated to play a role in signaling at the presynapse of the NMJ in Drosophila and therefore could be similarly affecting signaling at the presynapse in the brain (Pan and Broadie, 2007; Pan et al., 2008; Banerjee et al., 2010).

The role of DmGluRA in cognition has been previously demonstrated in studies of Drosophila models of Fragile X syndrome and Alzheimer's disease. Fragile $\mathrm{X}$ is the leading inherited cause of intellectual disability and the leading known genetic cause of ASD. A fly model is based on loss of the of the fly $d f m r 1$ gene, the ortholog of the human FMR1 gene, whose lack of expression leads to Fragile X syndrome. The Fragile X fly model has several behaviors in common with human symptoms including impairments in social interactions (Dockendorff et al., 2002) and cognitive impairments (McBride et al., 2005). Pharmacological blockade of the DmGluRA protein activity was able to rescue social interaction, immediate-recall memory and short-term memory in the Fragile $\mathrm{X}$ model representing the first time pharmacologic treatment rescued social impairments in an animal model of autism or memory impairments in an animal model of intellectual disability (Rubin, 1999b; McBride etal., 2005, 2012). Additionally, in this study, treatments initiated in development as well as those initiated in adulthood demonstrated efficacy in rescuing social interactions and memory.

DmGluRA has also been implicated in having a role in a Drosophila model of Alzheimer's disease that is based on mutations of the presenilin gene (McBride et al., 2010). The underlying nature of signaling alterations arising from the mutations in presenilin 1 or presenilin 2 genes that give rise to familial Alzheimer's disease (FAD) in humans are unclear (Saura et al., 2004; Walker et al., 2005; Qi-Takahara et al., 2005; Kumar-Singh et al., 2006; Sambamurti et al., 2006; De Strooper, 2007; Hardy, 2007; Isoo et al., 2007; Shen and Kelleher, 2007; Wolfe et al., 2007). Studies in model organisms indicate that the FAD-linked mutations lead to an impairment of presenilin 1 or presenilin 2 function (De Strooper, 2007; Kelleher and Purcell, 2008). This possibility suggests that some phenotypes associated with Alzheimer's disease, including age-onset cognitive loss, may be attributable to a reduction in overall presenilin protein activity levels. In the Drosophila Alzheimer's model young adult Drosophila (under 10 days of age, post-eclosion) have intact learning-during-training (LDT), immediate-recall memory and short-term memory, but have age dependent impairments in LDT and short-term memory at 30 days of age (McBride et al., 2010). Pharmacologic treatment with mGluR antagonists starting before cognitive impairments begin prevents cognitive impairment. Furthermore, treatment with mGluR antagonists starting after the onset of cognitive impairments reverses cognitive impairments in this model, indicating mGluR involvement in modulating synaptic plasticity well into adulthood (McBride et al., 2010). This indicates that in the Alzheimer's fly model, just as in the Fragile $\mathrm{X}$ fly model over active mGluR activity is contributing to memory impairment. More recently, under active mGluR activity has been implicated in phenotypes exhibited by tuberous sclerosis type 2 model mice (Auerbach et al., 2011). In spite of these findings, the involvement of DmGluRA in social interactions and memory in otherwise normal flies has remained unexplored in Drosophila. The purpose of this study was to examine the role of DmGluRA in social interactions and memory in Drosophila.

\section{RESULTS}

Social interaction can be examined in Drosophila in an ethologically relevant context by observing male courtship behavior directed toward female targets. Courting Drosophila males perform a characteristic sequence of behaviors: orienting toward and following the female, tapping her with his forelegs, vibrating one wing, licking her genitalia, and attempting to copulate (Bastock, 1955, 1956; Sturtevant, 1915). The percentage of time that the male spends performing any of these behaviors toward a target female during a defined period of time is referred to as the courtship index (CI; Siegel and Hall, 1979).

We first examined the ability of young adult (6-10 days posteclosion) homozygous null DmGluRA $A^{112}$ flies to perform naïve courtship with virgin female targets as well as the $D m G l u R A^{2 b}$, a precise excision control line. We found courtship behavior to be significantly impaired in the DmGluRA homozygous mutant flies, with the flies demonstrating almost no courtship activity (CIs of $3.2 \pm 0.4$ ), whereas the genetic background control flies demonstrated intact courtship behavior (CIs of $12.1 \pm 0.8$; Figure 1A). This demonstrated that the $\operatorname{DmGluRA}$ activity is required for social interaction since there was a significant impairment in naïve courtship behavior compared to the control strain.

The low courtship activity of the DmGluRA mutants prevented us from examining memory in the homozygous mutant mGluR flies. Therefore to explore a possible role of DmGluRA in memory, we examined courtship and memory in flies heterozygous for this mutation. To do this we crossed both the DmGluRA null mutant (DmGluRA ${ }^{112}$ ) and precise excision control $\left(D m G l u R A^{2 b}\right)$ lines to Oregon R flies. We found that in the heterozygous state there was still an impairment in social interaction of the DmGluRA ${ }^{112}$ heterozygous flies compared to the $D m G l u R A^{2 b}$ controls, $65.2 \pm 5.3$ vs $85.3 \pm 6.2$, again indicating a role for DmGluRA function in social interactions in Drosophila (Figure 1B). It should be noted that courtship in the OreR background is significantly higher than in the original background, mainly due to visual acuity differences in detecting motion, since the original background is white eyed. The OreR/OreR controls, the precise excision heterozygotes ( $\left.D m G l u R A^{2 b} / O r e R\right)$ controls and the mutant heterozygotes (DmGluRA $A^{112} /$ OreR) all had similar 


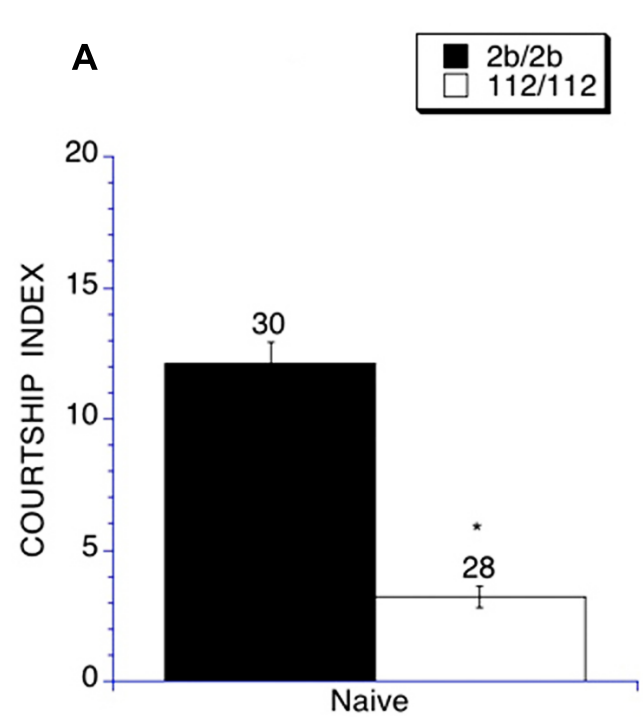

FIGURE 1 | Social interaction (Naïve courtship) is impaired in homozygous and heterozygous DmGluRA ${ }^{112}$ mutant flies.

Naïve courtship of DmGluRA, DmGluRA 112 homozygous mutant flies, control DmGluRA ${ }^{2 b}$ precise excision homozygous flies, DmGluRA 112 heterozygous mutant flies, control DmGluRA $2 b$ precise excision Filled black bars indicate control homozygous males (DmGluRA ${ }^{2 b}$ precise excision, $2 \mathrm{~b} / 2 \mathrm{~b}$ ) and open bars indicate DmGluRA ${ }^{112}$ homozygous mutant heterozygous flies and control OreR background flies were used. Panel (A)

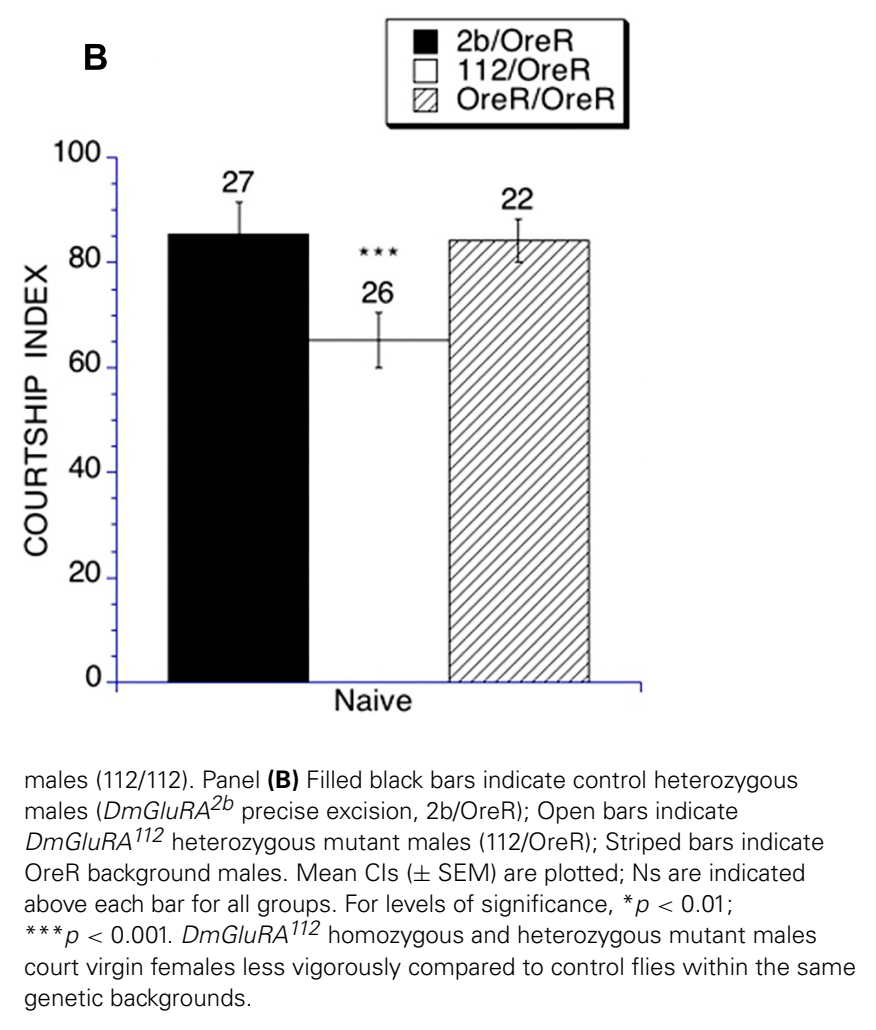

eye color. Furthermore, both control genotypes displayed similar levels of naïve courtship activity.

Although the heterozygous DmGluRA mutant flies displayed reduced naive courtship activity, they still retained enough courtship activity to examine learning and various forms of memory using the conditioned courtship memory paradigm, an associative memory paradigm. In conditioned courtship, a male fly learns to modify his courtship behavior after experience with an unreceptive female (Siegel and Hall, 1979; Hall, 1994). Virgin females generally respond to a courting male by mating. However, recently mated females are unreceptive, display rejecting behaviors toward advances made by the male and have an overlapping but altered pheromonal profile that naïve males find less provocative than that of virgin female targets (Ejima et al., 2007). Normally, naïve male paired with a mated female target will initially court her, but his courtship activity soon decreases. This LDT is quantified, by comparing the CI during the first $10 \mathrm{~min}$ to the $\mathrm{CI}$ of the last 10 min period of a $1 \mathrm{~h}$ pairing with a previously mated female. In this paradigm wild-type flies typically show $\mathrm{a} \geq 40 \%$ decrease in courtship activity (Joiner and Griffith, 1997; Kane et al., 1997). Hence, LDT is a form of behavioral plasticity but is distinct and separate from courtship suppression assayed after training, which is a form of associative memory (Tompkins et al., 1983; Ackerman and Siegel, 1986). When a male is paired with a virgin female after $1 \mathrm{~h}$ pairing experience with a mated female, his courtship remains depressed for 2-3 h (Siegel and Hall, 1979). This effect is not a general suppression of all courtship activity, because trained males do not modify their courtship of other pheromonally distinct targets (Ejima et al., 2005; Siwicki et al., 2005). After training with a mated female, memory is measured as a decrease in CI toward virgin females in trained males relative to naïve (sham trained) controls.

In Drosophila, five phases of memory have been elucidated by a combination of genetic and pharmacological dissection. There is an immediate-recall memory (immediate memory) at 0 2 min after training, short-term memory out to $1 \mathrm{~h}$ post-training, medium-term memory out to $6 \mathrm{~h}$ post-training, anesthesiaresistant memory out to 2 days post-training, and long-term memory lasting up to 9 days after training that appears to be dependent on protein synthesis (Skoulakis and Grammenoudi, 2006). Intact short-term memory is dependent on intact immediate recall. However, immediate recall and short-term memory are distinct from LDT. Therefore, intact memory can occur without LDT, and LDT can occur without post-training memory (Joiner and Griffith, 1997; Kane et al., 1997; McBride et al., 2005). Hence, in this study we chose to examine LDT, immediate-recall memory, short-term memory and long-term memory.

To assess LDT, a male fly was placed in a training chamber with a previously mated female for $1 \mathrm{~h}$, and the amount of time the male spent courting in the initial 10 min interval was compared with the time spent engaged in courtship in the final $10 \mathrm{~min}$ interval. Heterozygous DmGluRA $A^{112}$ mutants display impaired LDT as young adults (Figure 2), similar to what he been previously observed in older DmGluRA ${ }^{112}$ mutant flies at 30 days of age (McBride et al., 2010). In contrast heterozygous DmGluRA $A^{2 b}$ controls and the OreR/OreR controls displayed intact LDT. This demonstrates a requirement for DmGluRA function in LDT. 


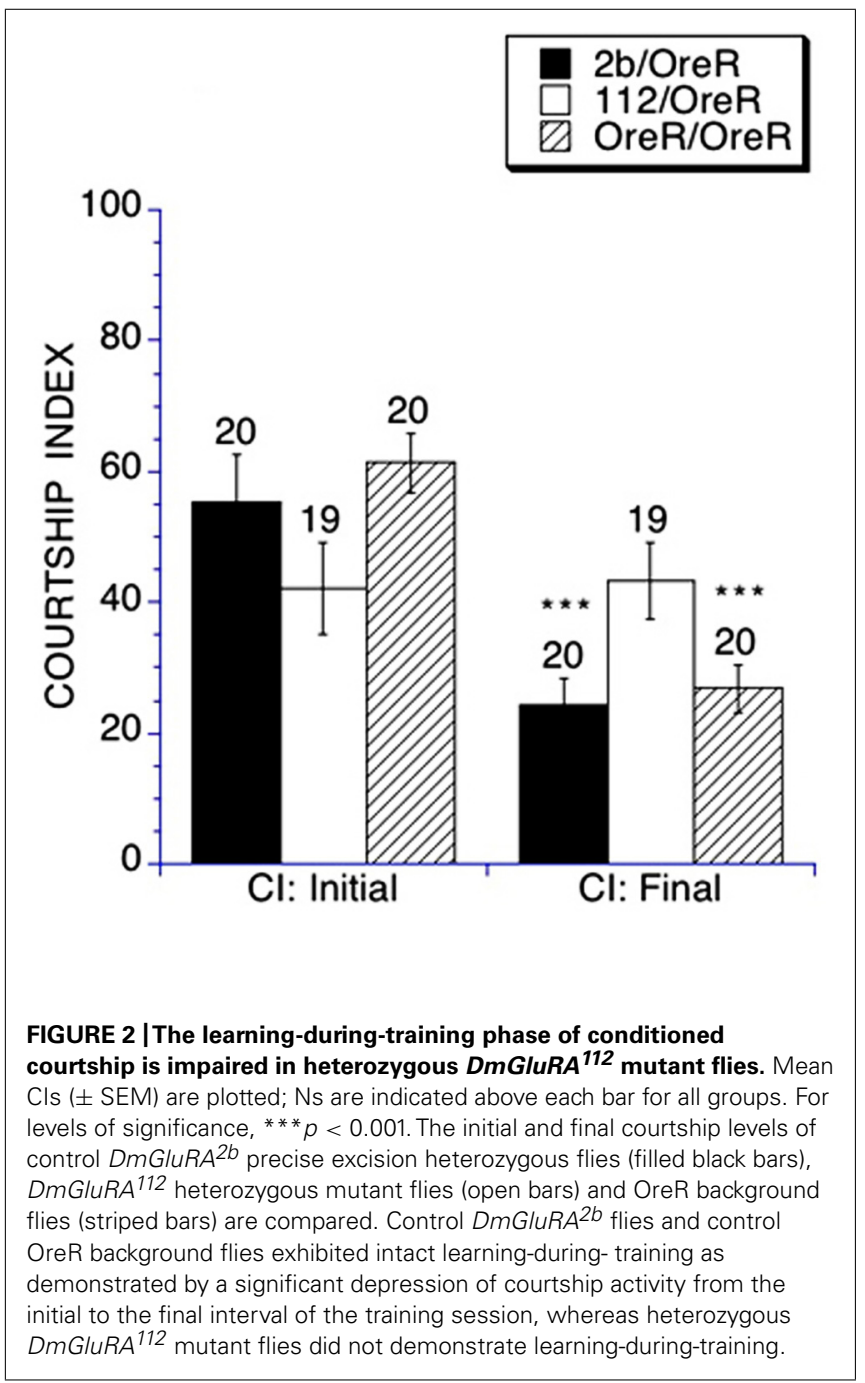

To assess immediate-recall memory, a male fly was placed in a training chamber with a previously mated female for $1 \mathrm{~h}$, and subsequently paired with a virgin female within 2 min of completing training. A lower CI compared to naïve trained (untrained) flies is indicative of memory. Heterozygous DmGluRA ${ }^{112}$ mutants display impaired immediate-recall memory as young adults, as they are not able to suppress their courtship upon subsequent pairing with a virgin female target (Figure 3). In contrast heterozygous $D m G l u R A^{2 b}$ controls and the OreR/OreR controls displayed intact immediate-recall memory. This demonstrates a requirement for DmGluRA function in immediate-recall memory.

To assess short-term memory, a male fly was placed in a training chamber with a previously mated female for $1 \mathrm{~h}$, and subsequently paired with a virgin female $60 \mathrm{~min}$ after completing training. A lower CI compared to naïve-trained flies is indicative of memory. Heterozygous DmGluRA ${ }^{112}$ mutants do not demonstrate a suppression of their courtship upon subsequent pairing with a virgin female target, therefore they do not demonstrate short-term memory (Figure 4). In contrast heterozygous DmGluRA ${ }^{2 b}$ controls and the OreR/OreR controls displayed a suppression of courtship after training and therefore demonstrated short-term memory. This

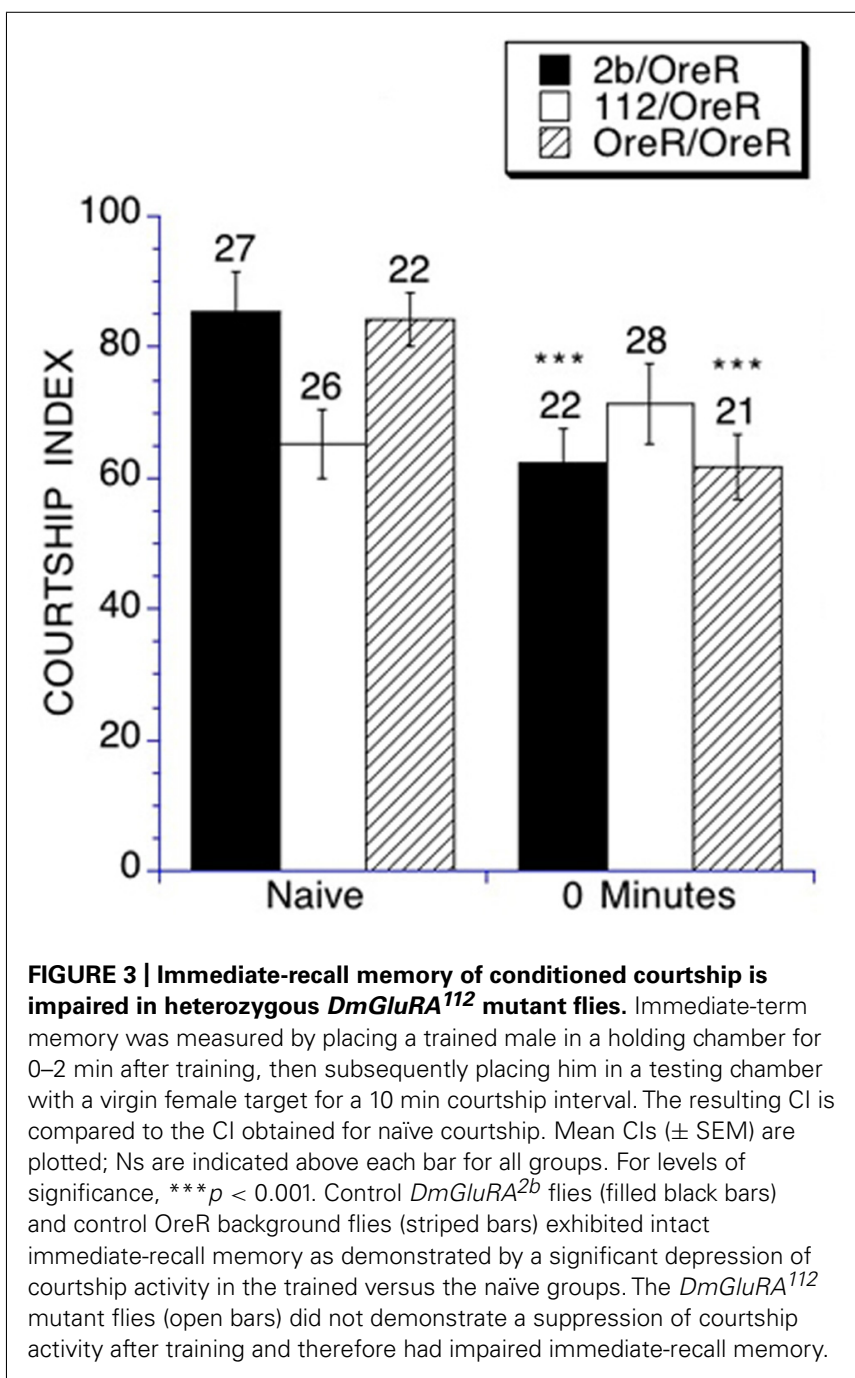

demonstrates a requirement for DmGluRA function in short-term memory.

Finally we examined if DmGluRA function was required for long-term memory (McBride et al., 1999; Banerjee et al., 2010). To assess long-term memory, a male fly was placed in a training chamber containing food with a previously mated female for $7 \mathrm{~h}$, and subsequently paired with a virgin female 4 days after completing training (McBride et al., 1999; Banerjee et al., 2010). Again, a lower CI compared to sham trained (naïve-trained) flies is indicative of memory. Heterozygous DmGluRA $A^{112}$ mutants do not demonstrate a suppression of their courtship upon subsequent pairing with a virgin female target, therefore they do not demonstrate longterm memory (Figure 5). In contrast heterozygous DmGluRA ${ }^{2 b}$ controls and the OreR/OreR controls displayed a suppression of courtship after training and therefore demonstrated long-term memory. This demonstrates a requirement for DmGluRA function in the formation of long-term memory.

To ensure that the decreased courtship activity of the homozygous and heterozygous DmGluRA ${ }^{112}$ mutants was not the result of specific impairment in not being able to complete the various phases of courtship, we measured the percentage of flies 


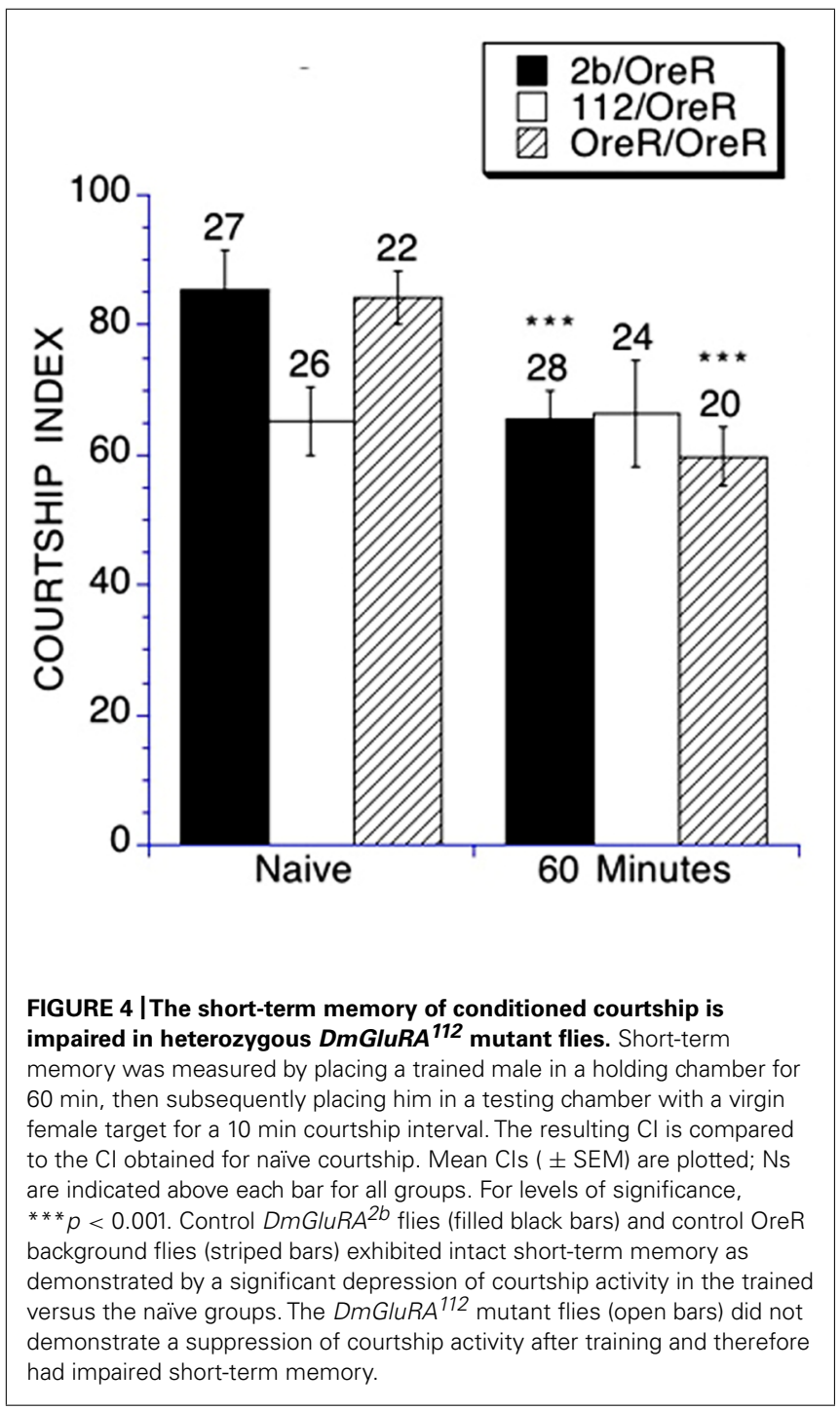

that progressed through the stages of courtship. The homozygous DmGluRA ${ }^{112}$ mutants demonstrated the ability to progress through all of the stages of courtship in a 10 min testing period. Both the homozygous DmGluRA $A^{112}$ mutants and the homozygous control precise excision $D m G l u R A^{2 b}$ had a significantly lower percentage of flies progressing to the licking/attempted copulation stage compared to the flies that were crossed to the OreR background ( $p<0.05$ by chi square). However, the DmGluRA $A^{112}$ mutants and the homozygous control precise excision $D m G l u R A^{2 b}$ did not differ from each other in the percentage of flies that reached this final step (Figure 6A). Both of the heterozygous lines as well as the OreR background control reached similar percentages of achieving all stages of courtship (Figure 6A). Since both the homozygous and heterozygous DmGluRA ${ }^{112}$ mutant flies were observed to be capable of performing all of the steps of courtship, the lack of courtship activity does not appear to be secondary to some type of impairment that is rendering them incapable of completing all of the steps of courtship behavior. To ensure that the decreased courtship activity of the homozygous

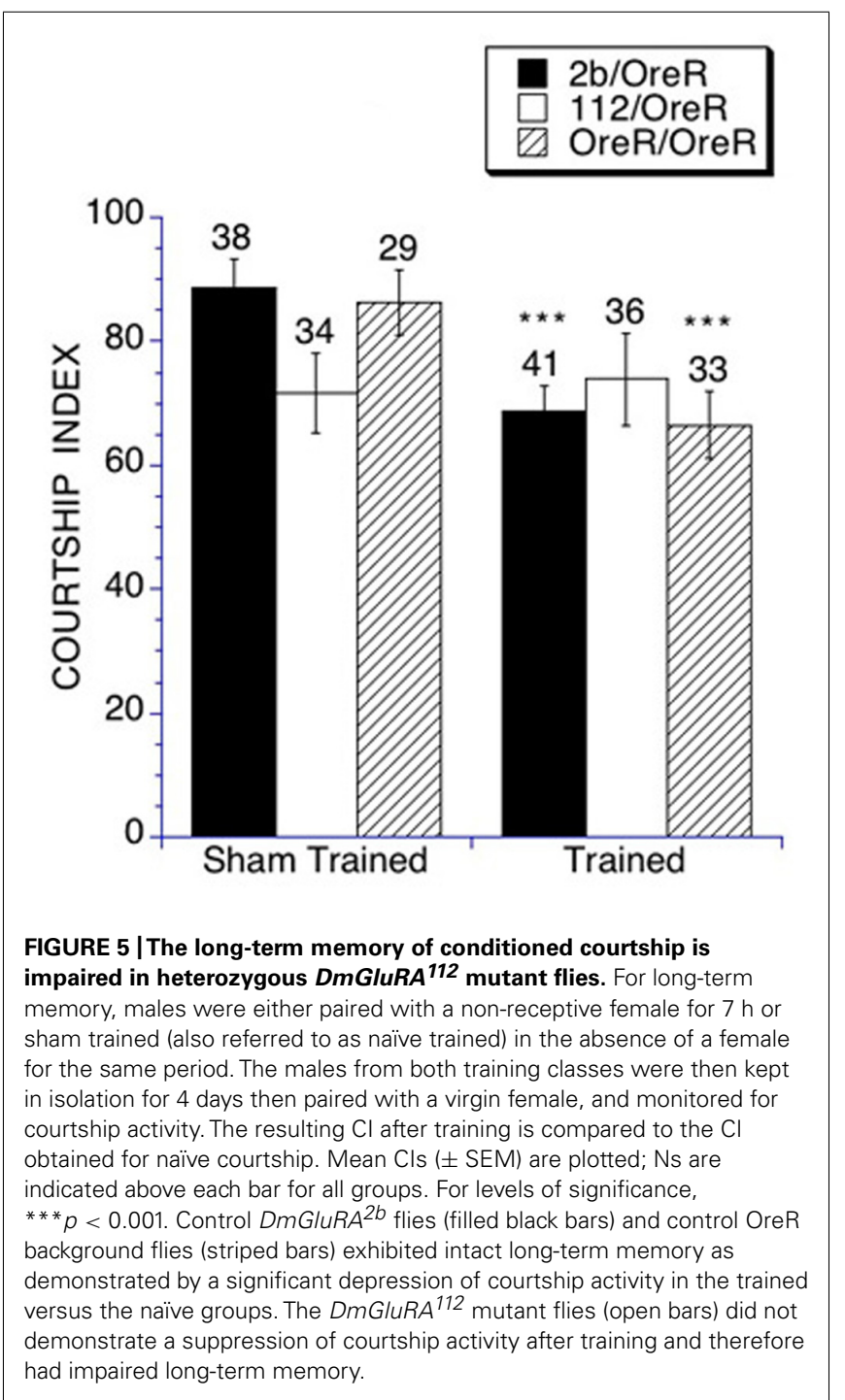

and heterozygous DmGluRA ${ }^{112}$ mutants was not the result of locomotor activity impairments, we examined locomotor function in the dishes utilized for the conditioned courtship testing (McBride et al., 2005, 2010). We did not find differences in spontaneous line crossing between homozygous or heterozygous DmGluRA ${ }^{112}$ mutant flies vs homozygous or heterozygous $D m G l u R A^{2 b}$ control flies or the OreR/OreR control flies (Figure 6B). Additionally neither the homozygous or heterozygous flies in any of the genotypes displayed gross impairments in olfaction or vision (Figures 6C,D).

\section{DISCUSSION}

Although a role for mGluRs signaling is well established in memory processes in mammals, the role of the only Drosophila mGluR, $\operatorname{DmGluRA}$, has remained relatively unstudied with regard to social interaction or memory (Serajee et al., 2003; Mukherjee and Manahan-Vaughan, 2012). The expression of DmGluRA in the Drosophila brain is in areas critically involved in social behavior and memory including the ALs and the MBs, thus may contribute 


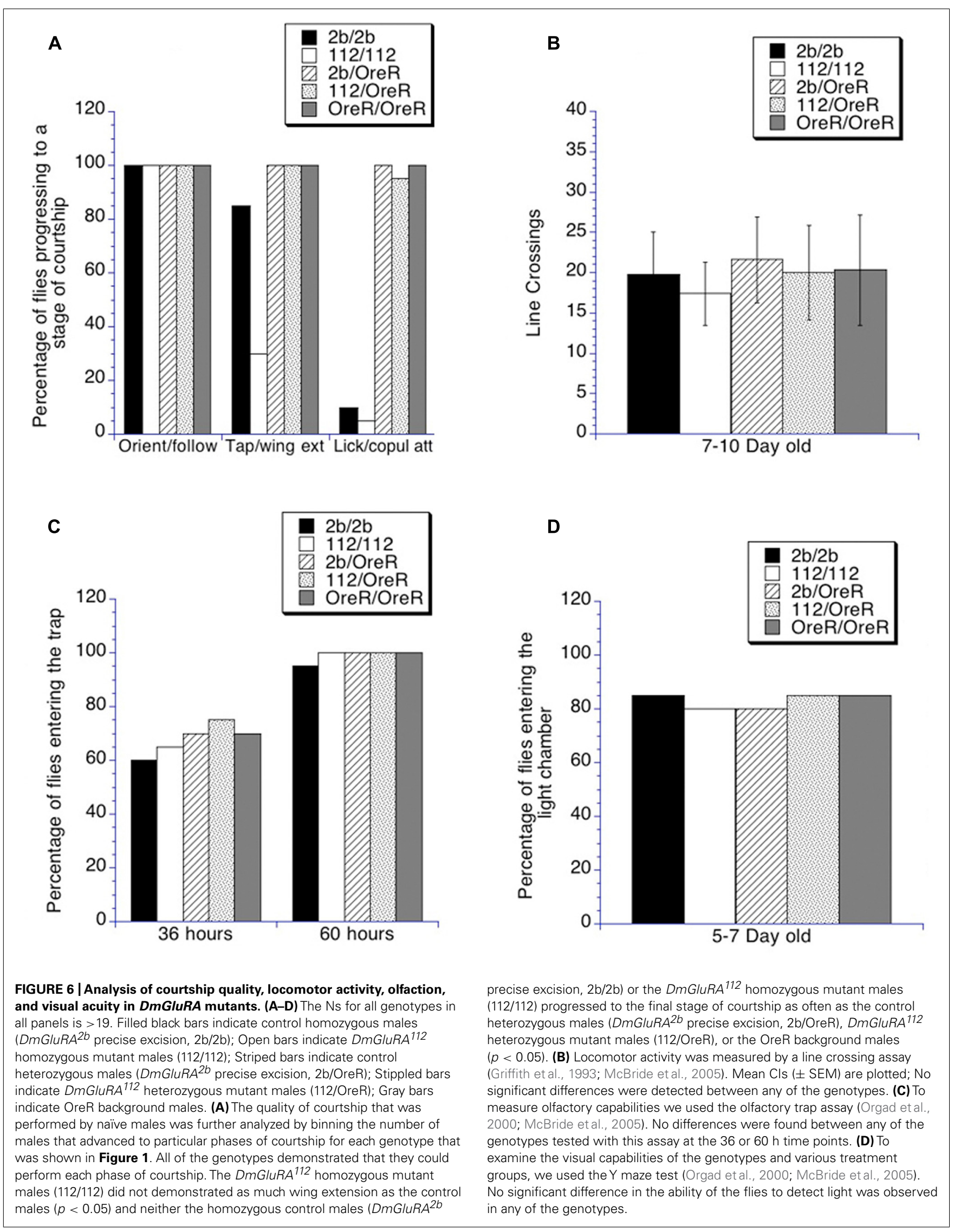


to these behavioral and cognitive processes (Ramaekers et al., 2001; Pan and Broadie, 2007; Pan et al., 2008).

Herein, we demonstrate that the DmGluRA activity is required for social interaction. We found that in the homozygous and heterozygous DmGluRA $A^{112}$ mutants have impairments in social behavior. This data fits well with the previous finding that a mutation in mGluR8 is linked to autism in humans (Serajee et al., 2003). Heterozygous DmGluRA ${ }^{112}$ mutants display impaired learning during training as young adults, thereby demonstrating a requirement for DmGluRA function in LDT, which may be analogous to working memory in mammals. Also heterozygous DmGluRA ${ }^{112}$ mutants display impairments in immediate-recall-memory, shortterm memory, and long-term memory as young adults. These findings fit well with the known role of mGluRs in short-term and long-term memory formation in mammals (Mukherjee and Manahan-Vaughan, 2012). In our study the deficits in social behavior and memory did not appear to be caused by impairments in vision, olfaction, locomotion, or the capability to perform complicated coordinated motor tasks such as copulation or flight, thus DmGluRA function appears to be specific for social and cognitive tasks.

This study illustrates an evolutionarily conserved role of the mGluRs in synaptic plasticity and memory formation which is an important finding in the context of using lower organisms to model cognitive diseases such as Fragile X or Alzheimer's disease. Indeed, these are two disease models where antagonizing the Drosophila DmGluRA has been demonstrated to rescue social and/or memory impairments (McBride et al., 2005, 2010; Choi etal., 2010). It was in the Drosophila model of Fragile X that pharmacological blockage of DmGluRA protein function was first demonstrated to rescue social interaction, immediate-recall memory and short-term memory representing the first time pharmacologic treatment rescued social impairments in an animal model of autism or memory impairments in an animal model of intellectual disability (Rubin, 1999b; McBride et al., 2005, 2012). Additionally, it was demonstrated that treatments initiated in development as well as in adulthood demonstrated efficacy in rescuing social interactions and memory. The finding that adulthood treatments could ameliorate phenotypes associated with developmental disorders was paradigm shifting (Rubin, 1999a; State, 2010) and has now been demonstrated in other models of developmental disorders (Rubin, 1999c; Li et al., 2005; Guy et al., 2007). This strategy of decreasing mGluR activity to rescue cognition in the Fragile $\mathrm{X}$ model was later confirmed in mouse model of Fragile $\mathrm{X}$ by genetic and pharmacologic manipulation (Yan et al., 2005; Dolen et al., 2007; Choi et al., 2011) and has met with some early success in trials with Fragile X patients (Jacquemont et al., 2011). This demonstrates the important role of DmGluRA function not just in development, but also in adulthood, warranting additional studies.

In Drosophila or cell culture models of Alzheimer's disease antagonizing mGluRs has been efficacious in rescuing phenotypes associated with the models including cognitive impairment and Abeta secretion (Kim et al., 2010; McBride et al., 2010). Furthermore, both agonist and antagonists of mGluRs are under development for the treatment of depression in humans (Chaki et al., 2012; Mukherjee and Manahan-Vaughan, 2012). The current work along with the extensive mammalian literature on the involvement of mGluRs in memory illustrate that caution should be observed when looking at the mGluRs as receptors to modulate for the rescue of disease specific symptoms, because they may have unwanted effects on other aspects of cognition.

At first pass our results demonstrating that reduction of DmGluRA activity negatively impacts social behavior and cognition may seem counterintuitive, because antagonism of this receptors signaling can enhance memory in specific disease models. First, we have previously found that treatment with mGluR antagonists does not enhance memory in wild-type flies, indeed they impair memory and social activity (McBride et al., 2005; Choi et al., 2010). Also, toward this point it is important to keep in mind the way the molecular signaling occurs during memory formation. DmGluRA is predominantly coupled to $\mathrm{Gi}$, thereby suppressing cAMP signaling. There is well documented literature in the fly field that indicates that cognition is impaired if cAMP levels are either too high, or too low. The $d n c$ mutant has severe memory impairments and results from too much cAMP. The $d n c$ mutation would be analogous to the DmGluRA mutants, with too much cAMP. In contrast the rut mutation leads to too little cAMP and also results in memory impairment (Skoulakis and Grammenoudi, 2006). This would be analogous to the fly models of Alzheimer's disease and fragile $\mathrm{X}$ syndrome, where the problem is too little cAMP and it is corrected by treatment with mGluR antagonists which should correct the cAMP to a level were normal memory can occur (McBride et al., 2005, 2010; Choi et al., 2010, 2011).

In conclusion, this work demonstrates that in Drosophila, just as in mammals, proper DmGluRA function is required for social behavior and various aspects of cognition including LDT, immediate-recall memory, short-term memory, and long-term memory.

\section{MATERIALS AND METHODS BEHAVIORAL TRAINING AND TESTING}

Virgin male flies were collected under ether anesthesia within $4 \mathrm{~h}$ of eclosion. Virgin XX, y, f (attached X) females were collected on the day of eclosion and kept in food vials in groups of 10-15. Flies were aged in a $12 \mathrm{~h}$ light/dark cycle before behavioral training and testing. All testing was performed during the relative light phase. Mated females were 5 days old and observed to mate with a male the night before training. The virgin females that were used as targets were 4 days old (Joiner and Griffith, 1997; McBride et al., 1999, 2005).

For courtship behavior testing, males of the appropriate genotypes were collected within $4 \mathrm{~h}$ of eclosion and kept in isolation before testing. All flies were kept in $12 \mathrm{~h} \mathrm{light/dark}$ cycles at $25^{\circ} \mathrm{C}$ and $50-75 \%$ relative humidity and were aged 6-10 days posteclosion before training. All male subjects were transferred to fresh control food the day before testing (McBride et al., 1999, 2005, 2010). Male flies were assigned to random groups for behavior training and testing, which was performed blind (Siegel and Hall, 1979; Kane et al., 1997; McBride et al., 1999). The total amount of time a male was engaged in courtship activity while paired with an unanesthetized target female during a test period of $10 \mathrm{~min}$ or until successful copulation occurred was scored. A CI was calculated as the percentage of total observation time spent courting (Siegel and Hall, 1979). Testing of naïve courtship, LDT, immediate-recall 
and short-term memory were performed as previously described (McBride et al., 1999, 2005). For the naïve courtship analysis, the male was sham trained for $1 \mathrm{~h}$ in the training chamber without the addition of the female. The male was then transferred to the mating chamber containing a virgin female. Males were monitored for courtship activity that included orienting, following of the female, wing extension and vibration, tapping of female with his foreleg, genital licking and attempted copulation for a period of $10 \mathrm{~min}$, or until copulation occurred.

Measurement of immediate-recall was made by pairing a naïve male with a non-receptive female for a single $1 \mathrm{~h}$ training session and then placing him in a second chamber with a receptive female within 2 min of completing training. Short-term memory was assessed by taking a male that had been trained with a non-receptive female for $1 \mathrm{~h}$ and placing him in isolation for $1 \mathrm{~h}$ before pairing with a virgin, receptive, female. At least 16 animals were tested for each genotype during analyses of naïve courtship, learning during training, immediate recall, short-term memory, and long-term memory.

The training paradigm for assessment of long-term memory is derived from McBride et al. (McBride et al., 1999; Banerjee et al., 2010). Males were paired with a non-receptive female for seven continuous hours and then kept in isolation for 4 days before testing. Sham-trained males were treated identically, except for the exposure to the training female. The observers were blind to the genotypes of the animals for all courtship studies (Banerjee et al., 2010; Sidyelyeva et al., 2010). Locomotor, olfaction, and visual acuity testing was done as in the study by McBride et al. (Griffith et al., 1993; McBride et al., 2005; Orgad et al., 2000).

\section{Drosophila STRAINS}

The Drosophila strains were cultured as in the study by McBride et al. (2005). The DmGluRA mutant and control lines used during this study are white eyed and have been previously described and utilized, they are the previously described null allele of DmGluRA $\left(D m G l u R A^{112}\right)$ and precise excision wild-type allele $\left(D m G l u R A^{2 b}\right)$ that provides an appropriate background control for the null allele (Bogdanik et al., 2004). Heterozygous versions of the mutant and control were obtained by crossing males to Oregon $\mathrm{R}$ virgin females. Heterozygous DmGluRA ${ }^{112}$ and $D m G l u R A^{2 b}$ F1 males were selected from the resultant progeny (McBride et al., 2010). The DmGluRA locus is on the 4th chromosome.

\section{REFERENCES}

Ackerman, S. L., and Siegel, R. W. (1986). Chemically reinforced conditioned courtship in Drosophila: responses of wild-type and the dunce, amnesiac and don giovanni mutants. J. Neurogenet. 3, 111-123.

Auerbach, B. D., Osterweil, E. K., and Bear, M. F. (2011). Mutations causing syndromic autism define an axis of synaptic pathophysiology. Nature 480, 63-68.

Banerjee, P., Schoenfeld, B. P., Bell, A. J., Choi, C. H., Bradley, M. P., Hinchey, P., et al. (2010). Short- and long-term memory are modulated by multiple isoforms of the fragile $\mathrm{X}$ mental

\section{STATISTICAL ANALYSES}

Courtship index of tested males were subjected to arcsin square root transformations to approximate normal distributions since not all of the sets of data were normal distributions, as is common in conditioned courtship data sets (Joiner and Griffith, 1997; McBride et al., 1999, 2005). For statistical comparison between the genotypes and treatments, Two Way ANOVA was used for genotype and treatment, with genotype resulting in a $p$ value of 0.0001 and treatment resulting in a $p$ value of 0.0001 . The interaction $p$ value was 0.0001 . The post hoc analysis used for the comparison for the figures was the Bonferroni analysis (Villella and Hall, 1996; McBride et al., 1999, 2005; Ejima et al., 2007). For the figures we have placed asterisks according to the post hoc Bonferroni analysis since this is demonstrating memory or no memory within a specific genotype according to the provided experience (treatment) of the flies (Villella and Hall, 1996; McBride et al., 1999, 2005, 2010; Ejima et al., 2007). For line crossing experiments standard student $t$ test was used and for Figure 6 binning analysis, olfaction and vision studies chi squared analysis was performed (McBride et al., 2005). All statistics were performed using both Statview 3.0 and Prism 5.0.

\section{ACKNOWLEDGMENTS}

This work was supported by the FRAXA Research Foundation with grants to (Sean M. J. McBride, Catherine H. Choi, Thomas A. Jongens), National Institutes of Health (NIH) Grants GM086902 (Thomas A. Jongens), an Alzheimer's Disease Center pilot award from NIH Grant AG-10124 (Thomas A. Jongens), as well as an Alzheimer's Disease Research pilot award, a program of the American Health Assistance Foundation (Thomas A. Jongens). Thomas V. McDonald and Sean M. J. McBride were supported by a grant from Autism Speaks, and Eric Koenigsberg was supported by a summer research fellowship grant from The National Fragile X Foundation. The Albert Einstein College of Medicine MSTP grant funded Sean M. J. McBride. Sean M. J. McBride was also supported by an R25 MH060490 (Clinical Research Scholars Program in Psychiatry). We thank Evan Braunstein, Joseph Hinchey, Sean Campbell, Myles Akabas, Nancy Carasco, Peter Davies, and Kami Kim for critical comments during this project. We thank Oliver Schipper for help with fly husbandry. We thank Susan Horwitz and Charles Rubin for critical comments and generously providing space and resources.

DmGluRA regulates activitydependent synaptic facilitation and fine synaptic morphology. J. Neurosci. 24, 9105-9116.

Busto, G. U., Cervantes-Sandoval, I., and Davis, R. L. (2010). Olfactory learning in Drosophila. Physiology (Bethesda) 25, 338-346.

Chaki, S., Ago, Y., Palucha-Paniewiera, A., Matrisciano, F., and Pilc, A. (2012). mGlu2/3 and mGlu5 receptors: potential targets for novel antidepressants. Neuropharmacology 66, 40-52.

Choi, C. H., McBride, S. M., Schoenfeld, B. P., Liebelt, D. A., Ferreiro, D., Ferrick, N. J., et al.
(2010). Age-dependent cognitive impairment in a Drosophila fragile X model and its pharmacological rescue. Biogerontology 11, 347-362.

Choi, C. H., Schoenfeld, B. P., Bell, A. J., Hinchey, P., Kollaros, M., Gertner, M. J., et al. (2011). Pharmacological reversal of synaptic plasticity deficits in the mouse model of Fragile X syndrome by group II mGluR antagonist or lithium treatment. Brain Res. 1380, 106-119.

Davis, R. L. (1993). Mushroom bodies and Drosophila learning. Neuron 11, 1-14.

Davis, R. L. (2011). Traces of Drosophila memory. Neuron 70, 8-19. 
De Strooper, B. (2007). Loss-offunction presenilin mutations in Alzheimer disease. Talking Point on the role of presenilin mutations in Alzheimer disease. EMBO Rep. 8, 141-146.

Dockendorff, T. C., Su, H. S., McBride, S. M., Yang, Z., Choi, C. H., Siwicki, K. K., et al. (2002). Drosophila lacking dfmrl activity show defects in circadian output and fail to maintain courtship interest. Neuron 34, 973-984.

Dolen, G., Osterweil, E., Rao, B. S., Smith, G. B., Auerbach, B. D., Chattarji, S., et al. (2007). Correction of fragile X syndrome in mice. Neuron $56,955-962$.

Dujardin, F. (1850). Memoires sur le systeme nerveux des insectes. Ann. Sci. Nat. (Zool.) 14, 195-205.

Ejima, A., Smith, B. P., Lucas, C., Levine, J. D., and Griffith, L. C. (2005). Sequential learning of pheromonal cues modulates memory consolidation in trainer-specific associative courtship conditioning. Curr. Biol. 15, 194-206.

Ejima, A., Smith, B. P., Lucas, C., van der Goes van Naters, W., Miller, C. J., Carlson, J. R., et al. (2007). Generalization of courtship learning in Drosophila is mediated by cis-vaccenyl acetate. Curr. Biol. 17, 599-605.

Fan, H., Peck, O. M., Tempel, G. E., Halushka, P. V., and Cook, J. A. (2004). Toll-like receptor 4 coupled GI protein signaling pathways regulate extracellular signalregulated kinase phosphorylation and AP-1 activation independent of NFkappaB activation. Shock 22, 57-62.

Gatto, C. L., and Broadie, K. (2009). Temporal requirements of the fragile $\mathrm{x}$ mental retardation protein in modulating circadian clock circuit synaptic architecture. Front. Neural Circuits 3:8. doi: 10.3389/neuro.04.008. 2009

Griffith, L. C., Verselis, L. M., Aitken, K. M., Kyriacou, C. P., Danho, W., and Greenspan, R. J. (1993). Inhibition of calcium/calmodulin-dependent protein kinase in Drosophila disrupts behavioral plasticity. Neuron 10 , 501-509.

Guy, J., Gan, J., Selfridge, J., Cobb, S., and Bird, A. (2007). Reversal of neurological defects in a mouse model of Rett syndrome. Science 315 , 1143-1147.

Hall, J. C. (1994). The mating of a fly. Science 264, 1702-1714.

Hardy, J. (2007). Putting presenilins centre stage. Introduction to the Talking Point on the role of presenilin mutations in Alzheimer disease. EMBO Rep. 8, 134-135.

Huang, J., Mahavadi, S., Sriwai, W. Hu, W., and Murthy, K. S. (2006). Gi-coupled receptors mediate phosphorylation of CPI-17 and MLC20 via preferential activation of the PI3K/ILK pathway. Biochem. J. 396, 193-200.

Huang, L. Q., Rowan, M. J., and Anwyl, R. (1997). mGluR II agonist inhibition of LTP induction, and mGluR II antagonist inhibition of LTD induction, in the dentate gyrus in vitro. Neuroreport 8, 687-693.

Huang, L. Q., Rowan, M. J., and Anwyl, R. (1999a). Role of protein kinases $\mathrm{A}$ and $\mathrm{C}$ in the induction of mGluRdependent long-term depression in the medial perforant path of the rat dentate gyrus in vitro. Neurosci. Lett. 274, 71-74.

Huang, L., Rowan, M. J., and Anwyl, R. (1999b). Induction of longlasting depression by (+)-alphamethyl-4-carboxyphenylglycine and other group II mGlu receptor ligands in the dentate gyrus of the hippocampus in vitro. Eur. J. Pharmacol. 366, 151-158.

Isoo, N., Sato, C., Miyashita, H., Shinohara, M., Takasugi, N., Morohashi, Y., et al. (2007). Abeta42 overproduction associated with structural changes in the catalytic pore of gammasecretase: common effects of Pen-2 $\mathrm{N}$-terminal elongation and fenofibrate. J. Biol. Chem. 282, 1238812396.

Jacquemont, S., Curie, A., des Portes, V., Torrioli, M. G., Berry-Kravis, E., Hagerman, R. J., etal. (2011). Epigenetic modification of the FMR1 gene in fragile $\mathrm{X}$ syndrome is associated with differential response to the mGluR5 antagonist AFQ056. Sci. Transl. Med. 3, 64ral.

Joiner, M.-l. A., and Griffith, L. C. (1997). CaM kinase II and visual input modulate memory formation in the neuronal circuit controlling courtship conditioning. J. Neurosci. 17, 9384-9391.

Kahsai, L., Carlsson, M. A., Winther, A. M., and Nassel, D. R. (2012). Distribution of metabotropic receptors of serotonin, dopamine, GABA, glutamate, and short neuropeptide $\mathrm{F}$ in the central complex of Drosophila. Neuroscience 208, 11-26.

Kane, N. S., Robichon, A., Dickinson, J. A., and Greenspan, R. J. (1997). Learning without performance in PKC-deficient Drosophila. Neuron 18, 307-314.

Kelleher, A. D., and Purcell, D. F (2008). Controlling HIV infection:
HIV co-receptor gets the finger. Immunol. Cell Biol. 86, 641-642.

Kim, S. H., Fraser, P. E., Westaway, D., St George-Hyslop, P. H., Ehrlich, M. E., and Gandy, S. (2010). Group II metabotropic glutamate receptor stimulation triggers production and release of Alzheimer's amyloid(beta) 42 from isolated intact nerve terminals. J. Neurosci. 30, 3870-3875.

Kreibich, T. A., Chalasani, S. H. and Raper, J. A. (2004). The neurotransmitter glutamate reduces axonal responsiveness to multiple repellents through the activation of metabotropic glutamate receptor $1 . J$. Neurosci. 24, 7085-7095.

Kumar-Singh, S., Theuns, J., Van Broeck, B., Pirici, D., Vennekens, K. Corsmit, E., et al. (2006). Mean ageof-onset of familial alzheimer disease caused by presenilin mutations correlates with both increased Abeta42 and decreased Abeta40. Hum. Mutat. 27, 686-695.

Li, W., Cui, Y., Kushner, S. A., Brown, R. A., Jentsch, J. D., Frankland, P. W., et al. (2005). The HMG-CoA reductase inhibitor lovastatin reverses the learning and attention deficits in a mouse model of neurofibromatosis type 1. Curr. Biol. 15, 1961-1967.

McBride, S. M., Bell, A. J., and Jongens, T. A. (2012). Behavior in a Drosophila model of fragile X. Results Probl. Cell Differ. 54, 83-117.

McBride, S. M., Choi, C. H., Schoenfeld, B. P., Bell, A. J., Liebelt, D. A., Ferreiro, D., et al. (2010). Pharmacological and genetic reversal of age-dependent cognitive deficits attributable to decreased presenilin function. J. Neurosci. 30, 9510-9522.

McBride, S. M., Choi, C. H., Wang Y., Liebelt, D., Braunstein, E., Ferreiro, D., et al. (2005). Pharmacological rescue of synaptic plasticity, courtship behavior, and mushroom body defects in a Drosophila model of fragile X syndrome. Neuron 45, 753-764.

McBride, S. M., Giuliani, G., Choi, C., Krause, P., Correale, D., Watson, K., et al. (1999). Mushroom body ablation impairs short-term memory and long-term memory of courtship conditioning in Drosophila melanogaster. Neuron 24, 967-977.

Mukherjee, S., and Manahan-Vaughan, D. (2012). Role of metabotropic glutamate receptors in persistent forms of hippocampal plasticity and learning. Neuropharmacology 66 , 65-81.

Niswender, C. M., and Conn, P. J. (2010). Metabotropic glutamate receptors: physiology, pharmacology, and disease. Annu. Rev. Pharmacol. Toxicol. 50, 295-322.

Orgad, S., Rosenfeld, G., Greenspan, R. J., and Segal, D. (2000). courtless, the Drosophila UBC7 homolog, is involved in male courtship behavior and spermatogenesis. Genetics 155, 1267-1280.

Otani, S., Auclair, N., Desce, J. M., Roisin, M. P., and Crepel, F. (1999). Dopamine receptors and groups I and II mGluRs cooperate for long-term depression induction in rat prefrontal cortex through converging postsynaptic activation of MAP kinases. J. Neurosci. 19, 9788-9802.

Otani, S., Daniel, H., Takita, M., and Crepel, F. (2002). Long-term depression induced by postsynaptic group II metabotropic glutamate receptors linked to phospholipase $\mathrm{C}$ and intracellular calcium rises in rat prefrontal cortex. J. Neurosci. 22, 3434-3444.

Pan, L., and Broadie, K. S. (2007). Drosophila fragile $\mathrm{X}$ mental retardation protein and metabotropic glutamate receptor A convergently regulate the synaptic ratio of ionotropic glutamate receptor subclasses. J. Neurosci. 27, 12378-12389.

Pan, L., Woodruff, E. III, Liang, P., and Broadie, K. (2008). Mechanistic relationships between Drosophila fragile X mental retardation protein and metabotropic glutamate receptor A signaling. Mol. Cell. Neurosci. 37, 747-760.

Pascual, A., and Preat, T. (2001). Localization of long-term memory within the Drosophila mushroom body. Science 294, 1115-1117.

Pesavento, J. J., Bullock, C. R., LeDuc, R. D., Mizzen, C. A., and Kelleher, N. L. (2008). Combinatorial modification of human histone $\mathrm{H} 4$ quantitated by two-dimensional liquid chromatography coupled with top down mass spectrometry. J. Biol. Chem. 283, 14927-14937.

Qi-Takahara, Y., MorishimaKawashima, M., Tanimura, Y., Dolios, G., Hirotani, N., Horikoshi, Y., et al. (2005). Longer forms of amyloid beta protein: implications for the mechanism of intramembrane cleavage by gamma-secretase. J. Neurosci. 25, 436-445.

Ramaekers, A., Parmentier, M. L., Lasnier, C., Bockaert, J., and Grau, Y. (2001). Distribution of metabotropic glutamate receptor DmGlu-A in Drosophila melanogaster central nervous system. J. Comp. Neurol. 438, 213-225.

Rubin, M. (1999a). The healing power of prayer. J. Christ. Nurs. 16, $4-7$. 
Rubin, R. H. (1999b). Cytomegalovirus in the transplant population: current issues and future directions. Discussion and consensus points. Transpl. Infect. Dis. 1(Suppl. 1), 40-41.

Rubin, R. H. (1999c). Cytomegalovirus and transplantation: yesterday, today, and tomorrow. Transpl. Infect. Dis. 1, 87-88.

Sambamurti, K., Suram, A., Venugopal, C., Prakasam, A., Zhou, Y., Lahiri, D. K., et al. (2006). A partial failure of membrane protein turnover may cause Alzheimer's disease: a new hypothesis. Curr. Alzheimer Res. 3, 81-90.

Saura, C. A., Choi, S. Y., Beglopoulos, V., Malkani, S., Zhang, D., Shankaranarayana Rao, B. S., et al. (2004). Loss of presenilin function causes impairments of memory and synaptic plasticity followed by agedependent neurodegeneration. $\mathrm{Neu}$ ron 42, 23-36.

Serajee, F. J., Zhong, H., Nabi, R., and Huq, A. H. (2003). The metabotropic glutamate receptor 8 gene at $7 q 31$ : partial duplication and possible association with autism. J. Med. Genet. 40, e42.

Shen, J., and Kelleher, R. J. III. (2007). The presenilin hypothesis of Alzheimer's disease: evidence for a loss-of-function pathogenic mechanism. Proc. Natl. Acad. Sci. U.S.A. 104, 403-409.

Sidyelyeva, G., Wegener, C., Schoenfeld, B. P., Bell, A. J., Baker, N. E.,
McBride, S. M., et al. (2010). Individual carboxypeptidase D domains have both redundant and unique functions in Drosophila development and behavior. Cell. Mol. Life Sci. 67, 2991-3004.

Siegel, R. W., and Hall, J. C. (1979) Conditioned responses in courtship behavior of normal and mutant Drosophila. Proc. Natl. Acad. Sci. U.S.A. 76, 3430-3434.

Siwicki, K. K., Riccio, P., Ladewski, L., Marcillac, F., Dartevelle, L., Cross, S. A., et al. (2005). The role of cuticular pheromones in courtship conditioning of Drosophila males. Learn. Mem. 12, 636-645.

Skoulakis, E. M., and Grammenoudi, S. (2006). Dunces and da Vincis: the genetics of learning and memory in Drosophila. Cell. Mol. Life Sci. 63, 975-988.

State, M. W. (2010). The genetics of child psychiatric disorders: focus on autism and Tourette syndrome. Neuron $68,254-269$.

Sturtevant, A. H. (1915). Experiments on sex recognition and the problem of sexual selection in drosophila. J. Anim. Behav. 5, 351-366.

Tessier, C. R., and Broadie, K. (2012). Molecular and genetic analysis of the Drosophila model of fragile X syndrome. Results Probl. Cell Differ. 54, 119-156.

Tompkins, L., Siegel, R. W., Gailey, D. A., and Hall, J. C. (1983). Conditioned courtship in Drosophila and its mediation by association of chemical cues. Behav. Genet. 13, 565-578.

Villella, A., and Hall, J. C. (1996). Courtship anomalies caused by doublesex mutations in Drosophila melanogaster. Genetics 143, 331-344.

Walker, E. S., Martinez, M., Brunkan, A. L., and Goate, A. (2005). Presenilin 2 familial Alzheimer's disease mutations result in partial loss of function and dramatic changes in Abeta 42/40 ratios. J. Neurochem. 92 , 294-301.

Wolfe, N. D., Dunavan, C. P., and Diamond, J. (2007). Origins of major human infectious diseases. Nature 447, 279-283.

Yan, Q. J., Rammal, M., Tranfaglia, M., and Bauchwitz, R. P. (2005). Suppression of two major Fragile $\mathrm{X}$ Syndrome mouse model phenotypes by the mGluR5 antagonist MPEP. Neuropharmacology 49, 1053-1066.

Yu, D., Ponomarev, A., and Davis, R. L. (2004). Altered representation of the spatial code for odors after olfactory classical conditioning; memory trace formation by synaptic recruitment. Neuron 42, 437-449.

Yuskaitis, C. J., Mines, M. A., King, M. K., Sweatt, J. D., Miller, C. A., and Jope, R. S. (2010). Lithium ameliorates altered glycogen synthase kinase-3 and behavior in a mouse model of fragile $\mathrm{X}$ syndrome. Biochem. Pharmacol. 79, 632-646.

Zars, T., Fischer, M., Schulz, R. and Heisenberg, M. (2000).
Localization of a short-term memory in Drosophila. Science 288, 672-675.

Conflict of Interest Statement: The authors declare that the research was conducted in the absence of any commercial or financial relationships that could be construed as a potential conflict of interest.

Received: 10 August 2012; accepted: 26 April 2013; published online: 23 May 2013.

Citation: Schoenfeld BP, Choi RJ, Choi $\mathrm{CH}$, Terlizzi AM, Hinchey P, Kollaros $M$, Ferrick NJ, Koenigsberg E, Ferreiro D, Leibelt DA, Siegel SJ, Bell AJ, McDonald TV, Jongens TA and McBride SMJ (2013) The Drosophila DmGluRA is required for social interaction and memory. Front. Pharmacol. 4:64. doi: 10.3389/fphar.2013.00064

This article was submitted to Frontiers in Neuropharmacology, a specialty of Frontiers in Pharmacology.

Copyright (C) 2013 Schoenfeld, Choi, Choi, Terlizzi, Hinchey, Kollaros, Ferrick, Koenigsberg, Ferreiro, Leibelt, Siegel, Bell, McDonald, Jongens and McBride. This is an open-access article distributed under the terms of the Creative Commons Attribution License, which permits use, distribution and reproduction in other forums, provided the original authors and source are credited and subject to any copyright notices concerning any thirdparty graphics etc. 